\title{
TRIDAQ Systems in HEP Experiments at LHC Accelerator
}

\author{
Agnieszka Zagoździńska, Ryszard S. Romaniuk, Krzysztof T. Poźniak, and Piotr Zalewski
}

\begin{abstract}
The paper describes Trigger and Data Acquisition (TRIDAQ) systems of accelerator experiments for High Energy Physics. The background for physics research comprises assumptions of the Standard Model theory with basic extensions. On this basis, a structure of particle detector system is described, with emphasis on the following functional blocks: Front-End Electronics, Trigger and DAQ systems. The described solutions are used in the LHC experiments: ATLAS, ALICE, CMS and LHCb. They are also used in other accelerator experiments. Data storage and processing functionality is divided into two hardware systems: Trigger and Data Acquisition, that are dependent on each other. High input data rate impose relevant choices for the architecture and parameters of both systems. The key parameters include detailed system structure and its overall latency. Trigger structure is defined by the physics requirements and the storage capability of DAQ system. Both systems are designed to achieve the highest possible space and time resolution for particle detection. Trigger references are reviewed [1]-[39] as well as chosen accelerator research efforts originating in this country [40]-[83].
\end{abstract}

Keywords-TRIDAQ, trigger, RPC, CMS, HEP, electronic systems, detectors, front-end electronics, CERN, data acquisition systems, distributed measuring and control networks, photonic networks

\section{INTRODUCTION}

$\mathbf{H}$ IGH ENERGY PHYSICS (HEP) is aimed at research of fundamental particles and forces to explain the structure of matter. The Standard Model (SM) [1], [2] theory describes phenomena up to the scale of $10^{-20} \mathrm{~m}$. It is smaller than the scale of electroweak forces which are revealed at the distance range of $10^{-18} \mathrm{~m}$ to equalize with the EM fields. The SM groups fermions in generations, like the symmetry group $\mathrm{SU}(3)_{\mathrm{c}} \mathrm{SU}(2) \times \mathrm{U}(1)$ and assumes that particle masses are generated by the Higgs mechanism. There are some suggestions that the SM must be extended to contain newly discovered mechanisms. SM theory does not contain gravity forces that are comparable to electromagnetic for energy level greater than $100 \mathrm{GeV}$, via the masses of $\mathrm{W}^{+}, \mathrm{W}^{-}$and $\mathrm{Z}^{\circ}$ bosons. Without a new and more fundamental theory, most of questions cannot be resolved.

There are three main directions of theoretical speculations: technicolors models, supersymmetry theories and additional dimensions. Each of them differently explains a problem of fitting and unification of different kind of forces. Technicolor models [3] suggest that the Higgs boson is not a point particle

This work was supported in part by Polish Ministry of Science and Higher Education grant no. N N202 167440.

A. Zagoździńska, R. S. Romaniuk, and K. T. Poźniak are with Warsaw Univ. of Technology, and P. Zalewski is with National Center for Nuclear Research (e-mail: azago@cern.ch). but has an internal structure that will be discovered at energy $1 \mathrm{TeV}$. This theory is probably impossible to be mapped into realistic models. Supersymetric theory [4] assumes that each particle has a symmetrical shadow partner which spin value differs of $1 / 2$. It implies that each fermion with $1 / 2$ spin has its pair in boson with spin value 0 . Their names are created by adding s- prefix like selectron or squark. Each 0 or 1 spin boson has the corresponding fermion with spin value $1 / 2$. Their names are created by adding sufixes -ino like gluino or higgsino. The supersymetric family contains double number of fundamental particles with masses above $1 \mathrm{TeV}$. It is compatible with the Great Unification Theory (GUT) that establishes equalization of electroweak and electrostrong forces at the energy level about $10^{16} \mathrm{GeV}$. The GUT theory expands the SM but has not been experimentally acknowledged yet. The newest concept contains additional dimensions [5]. For electroweak forces up to $10^{-18} \mathrm{~m}$ scale spacetime is certainly four-dimensional. The previous considerations ignored gravity forces for interaction scale lower than $1 \mathrm{~mm}$. Additional dimensions for the gravity theory change the growth rate of gravitational constant. That results in strong influence of the gravity above the energy value of $1 \mathrm{TeV}$. Influence of the gravity allows to solve the fine tuning problem [6].

Current and planned future accelerator and non accelerator experiments are supposed to verify these theories or prove that SM is a fundamental theory and cannot be developed further. To explore interactions of scale lower than $10^{-18} \mathrm{~m}$, specialized detectors for the particle research are needed [7]. Most of them contain multilevel triggering system and expanded data acquisition system but differ by the used technology [8] Detectors must be sensitive to the presence of the particles having different charge, that are predicted by theory. It is possible by generating strong electromagnetic fields where the particles flight path is curved and registered. Electronic systems that trigger data registration must be fast, reliable and resistant to high radiation levels. Non accelerator experiments (Auger Project [9], DAMA [10]) explore particles produced in natural processes. Most of such detectors are hidden deep underground and are aimed to register extremely rare particles. This paper is focused on the trigger and data acquisition systems for the detector experiments in Large Hardron Collider (LHC) [11] at CERN.

\section{Detector System Structure}

Experiments requirements, budget and current technical capabilities define the detectors size and structure. ATLAS [12] and CMS [13] detectors in LHC are designed to investigate 


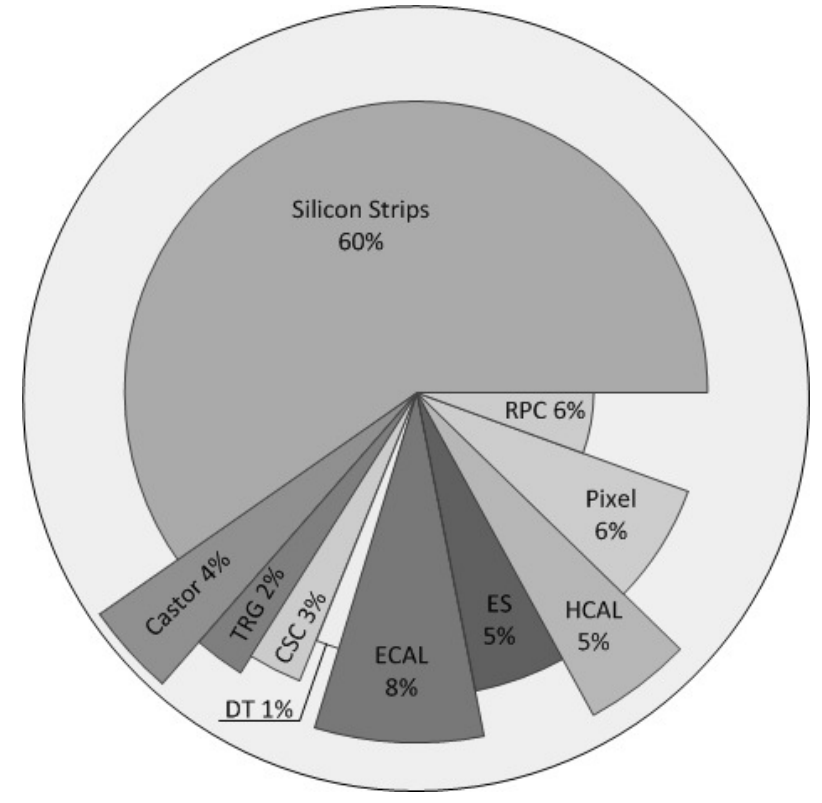

Fig. 1. Example of the data distribution produced in the different CMS sub-systems for proton-proton collisions.

the products of particle collisions. Both are general purpose detectors that have recently discovered the Higgs boson, and are expected to discover extra dimensions, as well as particles responsible for dark matter existence. Successive layers in the detectors are: the tracker, EM calorimeters, hadronic calorimeters and muon systems. General structure of the detectors is similar but all the systems and subsystems have different internal structure. The most visible difference is in the detectors size. ATLAS weight is only 7000 ton and the volume is $20000 \mathrm{~m}^{3}$ while the CMS is 12500 ton and about 3800 $\mathrm{m}^{3}$. Detector technology is not the only difference between the experiments. Collision rate and the rate of registered data determines the number of channels and their density. In $\mathrm{LHCb}$ detector [14], [15], which is a stationary target, the luminosity is low and only one side of the collision is investigated. The number of channels is lower than in most of the detectors in LHC, but the amount of registered data is higher. The input rate to HLT (high level trigger) is about $1 \mathrm{MHz}$ in $\mathrm{LHCb}$, while for CMS it is reduced to $100 \mathrm{kHz}$.

This task is performed by a multilevel triggering and data acquisition system realized using fast, custom made electronics. Much slower, high level trigger system, implemented in computer farms, receives a reduced amount of the data. The input rate is different in each part of the detector and subdetectors. In CMS 75\% from 100 million electronic channels comes from the tracker sub-detector. The particles are detected by the silicon strips and pixels. The pixel readouts supply 6000 connections per square centimeter, that is 65 million pixels in the whole sub-detector. $65 \%$ of the output signals is produced by the pixel readout in the tracker sub-detector. The average event sizes, for different CMS subsystems, for p-p collisions, are shown in Fig. 1. Each shade of grey corresponds to the separate subsystem. Some detector chambers transmit more data than the others. That is marked as the length difference of some wedges. The whole system is designed for an event size of $1 \mathrm{MB}$, at the maximum luminosity.

\section{FRONT END ELECTRONICS}

Signals from the detector are collected by the front-end boards. The electronics connected directly to the detector must be sensitive to short current pulses and resistant to high level radiation. The detector response depends on the charge deposit, event rate and sensitiveness of the device. Its measurement precision is limited by the charge absorption in the detector and electronic noise. Signal charge for a given energy absorption is formed by many elementary excitations [18]. Increase of the measurement resolution improves the signal to background ratio but limits the maximum signal bandwidth. For this reason, fast rising signals are shaped as long as there is a spacing between the pulses. This can be difficult to obtain when the time interval between the collisions is low. If the beam is continuous, the distinction between bunch crossings is possible only with the time measurement. Most of the detectors have particular drift time that limits the time resolution. For Drift-tubes detectors the minimum time resolution is $1 \mathrm{~ns}$, because location of the particle cannot be accurately determined. Better resolution is achieved in the Time Projection Chambers. Very high time resolution, up to 10-100ps, can be achieved only in the Time Of Flight (TOF) detectors. Such detectors are used e.g. in ALICE experiment [19]. The measurement resolution is also limited by the time dependency on amplitude. Its influence can be reduced by the use of constant fraction discriminators. The main disadvantage of this solution is the additional time delay. The amplitude can be compensated by the use of two channels of a Time to Digital Converter (TDC). Each of them is set for a different threshold [20]. In systems of the size comparable to ATLAS or CMS, the number of such modules is a few thousands. Their duplication would significantly increase the measurement time and cost. The alternative method is Time Over Threshold (TOT), where both leading edge and pulse width are measured in each channel. Such system is used in the Atlas Pixel Detector [21].

The amplified and shaped analog signals are converted to digital. This conversion process can be executed in various places of the processing chain. Depending on the conversion site, the systems can be divided into the following categories: analog readout and buffering, digital readout with analog buffering or both digital. In majority of the systems, the conversion is realized as soon as possible. Converters must provide sufficiently high level of the sensitivity and accuracy within a given budget. High resolution converters must work with low jitter clocks to avoid the additional source of noise. If the A/D converters have an insufficient speed or their power consumption is too high, analog buffers are used. Such buffers are used both in small storage systems, like sampling oscilloscopes, and in complex multichannel systems like the CMS tracker, Atlas calorimeter or LHCb trackers. Even if the buffers decrease the output rate, it can be still too high for what the Data Acquisition (DAQ) system can handle. The real life data rate cannot be estimated before the experiment is 


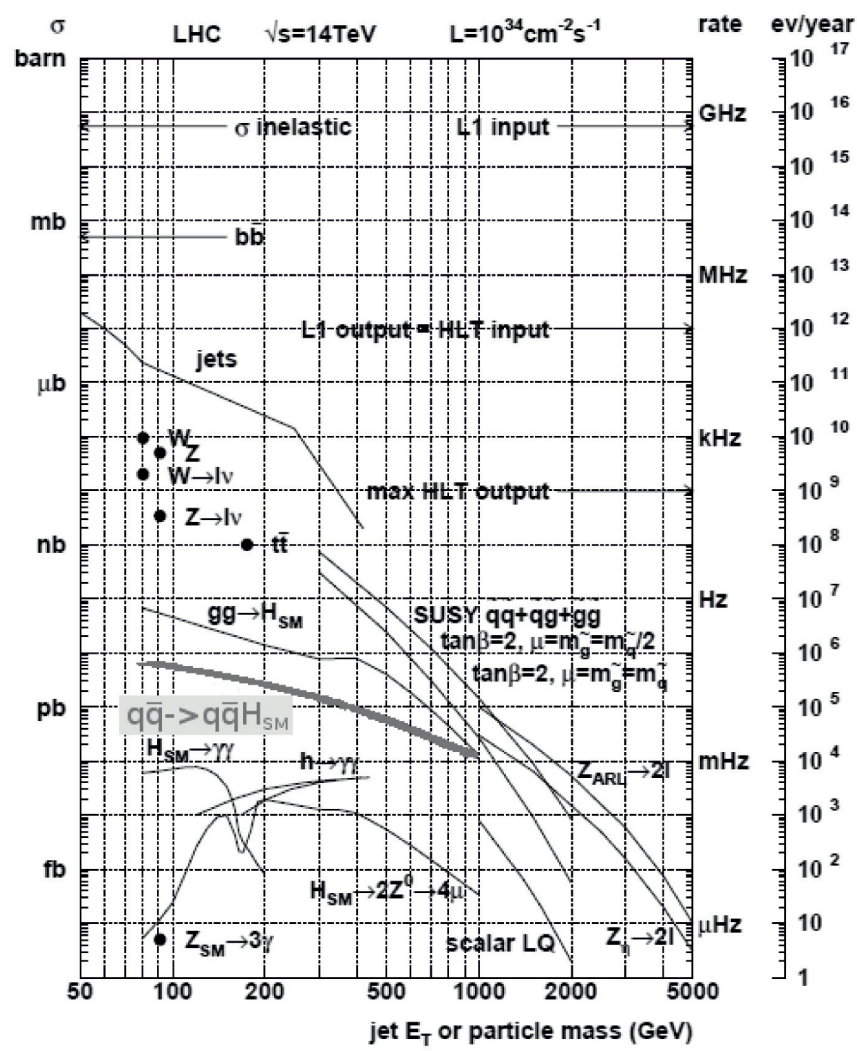

Fig. 2. Inclusive proton-(anti)proton cross sections $\sigma$ for basic physics processes. Interaction rates for the nominal luminosity are given on the righthand scale.

running. The estimation is based on physical simulations and experiences of the previous experiments. This can cause some problems in the hardware design. The buffers depth is limited by the cost and technical capabilities. If these parameters are too small there is a risk of the data overflows. In many systems the zero-suppression algorithm is implemented. That enables reduction of the data amount before it is sent to the trigger. Only non-zero data are transmitted to the readout system, synchronically to the global bunch-crossing clock. This method is regarded as the most efficient solution for the very high-rate future experiments such as SLHC [22] or CLIC [23].

\section{The Triggering System}

The trigger system analyzes events in regular intervals, in the collider experiments. The particles collide and interact during each bunch-crossing (BX). The method of the data selection is defined by the requirements of particular HEP experiment. The smaller granularity of the matter, the greater energy, luminosity and collision rate are required for the research. The rate was only $45 \mathrm{kHz}$ for LEP [24], $2.5 / 7.6 \mathrm{MHz}$ for Tevatron [25] and $40 \mathrm{MHz}$ for LHC. For high luminosity hadron colliders, each $\mathrm{BX}$ can provide up to 25 events that contain different number of muons, clusters etc. This rate could not be handled by any data acquisition system. The collision cross section $\sigma$ unit is the barn [b]. The barn describes the probability of interaction between particles in collider experiments for HEP. At LHC, the total proton-proton cross section is about $70 \mathrm{mb}$ [26], [27]. For a nominal collision energy, the frequency of particles generation (Fig. 2) is $70 \mathrm{~Hz}$ for Beauty quark $(0.7 \mathrm{mb}), 1 \mathrm{kHz}$ for $\mathrm{W} / \mathrm{Z}$ bosons $(200 / 60$ $\mathrm{nb}), 8 \mathrm{~Hz}$ for Top quark $(0.8 \mathrm{nb})$ and $0.3 \mathrm{~Hz}$ for Higgs $150 \mathrm{GeV}$ boson (30pb). This means that only small part of the generated data is useful for the analysis. To cut off an uninteresting physics information, the multilevel trigger system is used. It is realized with the use of signatures that contain parameters such as a threshold, amplitude or more complex data. The set of parameters is initially based on software simulation and calculation of muon tracks, energy deposits in the calorimeters, and track in the silicon detectors. Particles such as muons and electrons have clear signatures. A separation of a single lepton from the jets requires an analysis of particle showers. The trigger systems at the collider experiments are sensitive to the particles transverse momentum $\left(\mathrm{p}_{\mathrm{T}}\right)$.

The trigger system must be simple and selective, to follow the incoming data in the real time. The low level triggers are implemented using specialized electronics with fast FPGA chips [28]. The high level trigger is realized off line by computer farms. The final output rate is limited by an offline computing budget and storage capacity of the system. A deadtime is determined as a ratio between a time when the data acquisition system is busy and a total time. An important factor increasing the dead-time value is random distribution of the data. Most of the events occur within a short time window, then the fraction of the system busy time is much higher. Typically, the system is busy during the ADC conversion and storage time. In the system enhanced with an additional FIFO queue, the dead-time is reduced to the conversion time. The storage element must be large enough to follow the full data size during the system operating time. The effective trigger rate is reduced to a level that can be handled by the readout system. At the CDF Trigger System in Fermilab [29], the average deadtime is kept below 5\% [30]. At the BNL-E949 experiment in the Alternating Gradient Synchrotron for the Level-0 trigger, the online dead time was reduced from $4.0 \%$ to $1.7 \%$ [31]. At the CMS Level-1 trigger, for the maximum output rate of $100 \mathrm{kHz}$, the dead time is estimated to be below $1 \%$ [32].

Most of the HEP experiments contain a multilevel trigger system [33]. The triggering task can be divided to 2 (CMS), 3 (ATLAS and LHCb) or 4 levels (ALICE). The Level-0 Trigger is based on the hardware implementation with the use of FPGAs. The data cannot be processed longer than a few $\mu$ s, so selection algorithms have to be as simple as possible. The selection is aimed primarily at the identification of leptons. Analysis covers the low precision data from detectors like hadron and electromagnetic calorimeters for electrons $/ \gamma /$ jets or the muon chambers. Its results depend on the values of programmable parameters such as $\mathrm{p}_{\mathrm{T}}$ threshold and $\mathrm{BX}$ identification methods that are implemented. The high level trigger (HLT) performs more complex algorithms and can be busy for a few ms up to 10s. The algorithms are implemented in a software and hardware. The HLT is a selective process. At some experiments, its functionality can be divided into two levels: accessing to the part of the event (Level-1) and to the full event (Level-2). Both parts analyze the full precision 


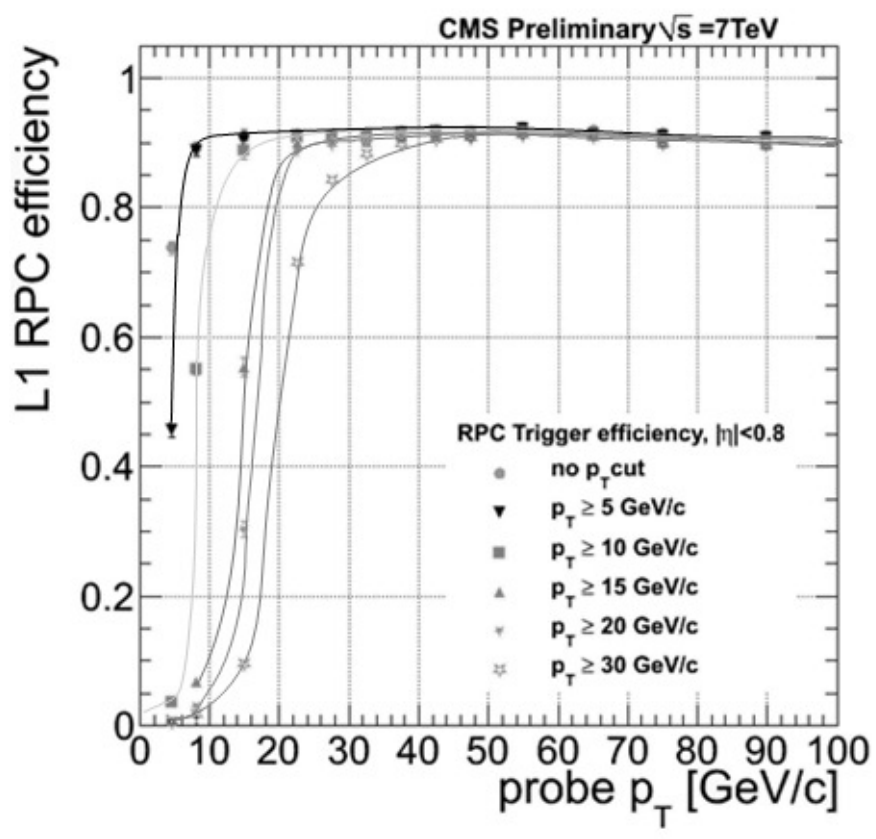

Fig. 3. RPC Trigger efficiency measured by the Tag \& Probe method for the barrel, Data: $2011 \mathrm{~B}$, probes with $\mathrm{p}_{\mathrm{T}}>20 \mathrm{GeV}$ from Z's, probes with $\mathrm{p}_{\mathrm{T}}$ $<20 \mathrm{GeV}$ from $\mathrm{J} / \psi$ 's [36].

and granularity information from the chambers. The HLT uses the topological variables and tracking information from inner detectors e.g. the ATLAS calorimeter trigger provides the information about the particles e, $\gamma, \tau$, jets and also about $\mathrm{E}_{\text {Tmiss }}, \Sigma \mathrm{E}_{\mathrm{T}}$ [34]. The triggering conditions can be imposed to reduce the jet background at low energy thresholds.

The difficulty for the trigger system is to decrease the data rate and to enable collection and data processing that are of interest for physics. Both aims cannot be achieved simultaneously. When the efficiency grows, the rejection of the physical background is worse and vice versa, the improved background rejection means a decrease of the efficiency. In contrast to the precision experiments, where the well known selections are used, the discovery experiments use the inclusive selections. In both cases the rejected data are lost and cannot be restored. Current efficiency achieved at the RPC PAC trigger at the CMS is about $92 \%$ in the barrel (Fig. 3) and $80 \%$ in the endcaps for $\mathrm{p}_{\mathrm{T}}$ greater than $20 \mathrm{GeV} / \mathrm{c}$. Maintenance of high efficiency with increasing LHC luminosity requires a continuous work on the trigger patterns optimization. The system efficiency is defined as a number of events that passed the selection ratio to a number of all events. There are passthrough triggers implemented in the HLTs, to select samples without any bias. At the L0 Trigger, the number of events without the selection is an unknown parameter. There are commonly two methods used to estimate the efficiency. The first one is a comparison between the orthogonal triggers. The second method is the experimental technique called "Tagand-Probe" [35]. In this method, a particle track is selected (Tag) and a complementary offline track is found (Probe). The trigger selection is applied on the probe. The capability of the rate is not unlimited. Some particles cannot reach the

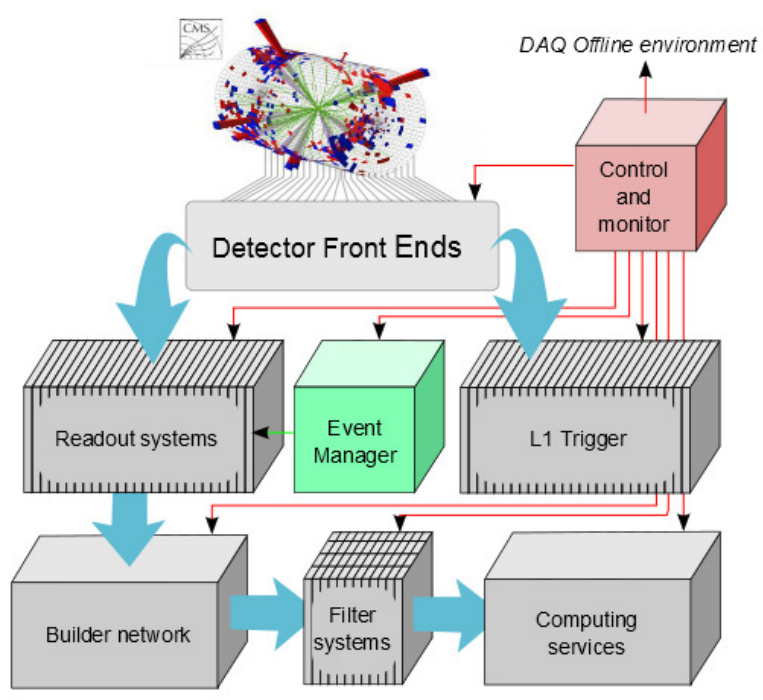

Fig. 4. Components of the DAQ system of CMS [38].

threshold level because their $\mathrm{p}_{\mathrm{T}}$ is underestimated. The error, in estimating $\delta \mathrm{p}_{\mathrm{T}} / \mathrm{p}_{\mathrm{T}}$, can reach $30 \%$ for the L0 Trigger, which has the worst spatial resolution.

\section{DATA ACQuisition System}

The detector system divides its functionality between the trigger and data acquisition systems. A dual architecture enables separation of the processing and storage functions. The trigger and DAQ parameters are shown in Tab. I. The data are buffered in the DAQ system. Data rate is decreased in the subsequent trigger levels from $\mathrm{MHz}$ to $\mathrm{kHz}$. Both systems work in a real-time mode where the latency is low and strictly determined. The DAQ system also builds and stores complete events from a segmented information from the detector. This process is called an event building and data logging. There are control, configuration and monitoring tools implemented in the DAQ. The basic parameters are, similarly to the Trigger System, the dead time and efficiency.

The DAQ system can be divided into four functional stages:

- a detector readout - collects the data in about 700 buffers (ATLAS, CMS),

- an event building stage - collects all the data corresponding to a single event,

- a selection stage - implements the HLT functionality in a processor farm,

- an analysis and storage - selected events are forwarded to the computing services for storage and analysis.

The general structure of the DAQ system at the CMS experiment is shown in Fig. 4. Two complementary systems enable the data flow from Detector Front Ends to processor farm. The Front-End Electronics is the first storage element in the system connected directly to the detecting units of each sub detector. There are approximately 700 modules in the CMS readout [39]. The data from Front-End Boards (FEB) are read and stored in the Readout System buffers. The storage is realized until the data can be sent further to a processor 
TABLE I

TRIGGER AND DAQ PARAMETERS [37]

\begin{tabular}{|c|c|c|c|c|c|c|c|}
\hline \multirow{2}{*}{$\begin{array}{c}\text { Detector } \\
\text { ALICE }\end{array}$} & \multirow{2}{*}{$\begin{array}{c}\overline{\text { Trigger }} \\
\text { levels } \\
4\end{array}$} & \multicolumn{2}{|c|}{$\overline{\mathrm{Rate}}[\mathrm{Hz}]$} & \multirow{2}{*}{$\begin{array}{c}\begin{array}{c}\text { Event size } \\
\text { [Byte] }\end{array} \\
\\
5 \times 10^{7} \\
2 \times 10^{6}\end{array}$} & \multirow{2}{*}{$\begin{array}{c}\begin{array}{c}\text { Readout } \\
{[\text { GB/s }]}\end{array} \\
2\end{array}$} & \multirow{2}{*}{$\begin{array}{c}\begin{array}{r}\boldsymbol{H} \\
{[M B / s]}\end{array} \\
1250 \\
200\end{array}$} & \multirow{2}{*}{$\begin{array}{c}\overline{\text { TTOut }} \\
\text { (Events } / \text { s) } \\
\left(10^{2}\right) \\
\left(10^{2}\right)\end{array}$} \\
\hline & & $\begin{array}{l}\text { L3 Pb-Pb } \\
\text { L3 p-p }\end{array}$ & $\begin{array}{l}500 \\
10^{3}\end{array}$ & & & & \\
\hline ATLAS & 3 & $\begin{array}{l}\text { L0 } \\
\text { L1 } \\
\text { L2 }\end{array}$ & $\begin{array}{c}10^{5} \\
3 \times 10^{3} \\
2 \times 10^{2}\end{array}$ & $1,5 \times 10^{6}$ & 4,5 & 300 & $\left(2 \times 10^{2}\right)$ \\
\hline CMS & 2 & $\begin{array}{l}\text { L0 } \\
\text { L1 }\end{array}$ & $\begin{array}{l}10^{5} \\
10^{2}\end{array}$ & $10^{6}$ & 100 & $\sim 1000$ & $\left(10^{2}\right)$ \\
\hline $\mathrm{LHCb}$ & 2 & $\begin{array}{l}\text { L0 } \\
\text { L1 }\end{array}$ & $\begin{array}{c}10^{6} \\
4 \times 10^{3}\end{array}$ & $3,5 \times 10^{4}$ & 35 & 70 & $\left(2 \times 10^{3}\right)$ \\
\hline
\end{tabular}

that analyze the event. The buffers are deep enough to wait until the decision from the L1 trigger is not ready. The Readout Unit supports the number of the FEBs. There are about 500 such Readout Columns. A positive trigger signal enables the further data transmission to the HLT processor farm. The transmission is through the Builder Network which is a large switch network with the $800 \mathrm{~Gb} / \mathrm{s}$ throughput. To provide the continuous data flow without traffic jams, the control signals are also sent through the switch. The Filter Systems process the events to realize the HLT algorithms. The selected events are finally stored for the offline processing. There are about 500 Filter Columns in the CMS. Each of them contains one Builder Unit and multiple Filter Units. The Builder creates a complete single event from the incoming data fragments. The single Filter Unit contains processors for the HLT algorithms. The filtered events, and small fraction of the rejected events, are sent to the Computing Services. The Event Manager is a unit responsible for the DAQ data flow. It centralizes the event management and synchronizes the overall system. A Control and Monitor unit is aimed to configure, control and monitor all system elements. It manages the interface to the DAQ offline environment. Such modular architecture of the DAQ system and its division into the four main tasks enables independent implementation and testing. It also requires a usage of an additional deep buffer. The buffers enable matching between all the operating stages, working at different rates.

\section{CONCLUSION AND SUMMARY}

The TRIDAQ systems must be consistent with the physics goals. At the LHC experiments the Trigger Systems are aimed to follow the rate of $200 \mathrm{kHz}$. The limitations are: the bandwidth, required efficiency and background rejection. The trigger rate during the design and commissioning is based on simulations containing large amount of the data with cross-section background. Initial analysis must include the information about the detector topology. The trigger must be flexible enough to cope with the real conditions that could be different than expected. The system must provide a channel masking to reject the channels that give much higher rate than expected. A typical solution is a programmable threshold implementation. The threshold level reduction increases the number of registered events. That is desirable until profits are higher than the offline computing cost. The data are analyzed simultaneously by the different triggering algorithms to follow the physics requirements. The trigger menu contains a set of parameters for each level of the trigger - L0, L1 and L2, that are grouped vertically to create a trigger chains. In an ideal situation, the triggered and stored data should overlap to save the storage space. There are two types of the TRIDAQ systems: dedicated to collect the data from accelerator and non accelerator experiments. The Trigger Systems for the HEP experiments are independent but complementary to the DAQ systems. Both are built with smaller blocks that can be designed and implemented separately. This is an important feature when the project is realized by groups from institutes scattered around the world. A specification of these systems is determined by the HEP predictions and experiences of the previous experiments. There is a clear trend of increasing a number of the collected data. The growth is a result of a few factors such as the increase of energy at accelerator experiments or the newest achievements in the magnets area. These factors enable better beam positioning and focusing that influence the reached luminosity and the number of triggered data. The current systems are designed to achieve the highest space and time resolution. The multiplication of the input channels has also an influence on the registered data size. All these factors seems to exist in the current and the nearest future experiments that are challenging to the TRIDAQ systems. This growth could be stopped if the registered event resolution exceeds the analysis and acquisition capability.

This work was presented during the Wilga 2012 Symposium on Electronic and Photonic Systems for High Energy Physics Experiments and Astronomy and in part published in the Wilga 2012 proceedings.

\section{REFERENCES}

[1] M. Schmaltz, "Physics beyond the standard model (Theory): Introducing the Little Higgs," Nuclear Physics B - Proceedings Supplements, vol. 117, pp. 40-49, 2003.

[2] C. Quigg, "The Standard Model (Electroweak Theory)," European School of High-Energy Physics, 2002.

[3] J. Andersen et al., "Discovering technicolor," The European Physical Journal Plus, vol. 126, no. 81, 2011. 
[4] P. Binétruy, Supersymmetry: Theory, Experiment, and Cosmology. Oxford University Press, 2006.

[5] Website http://cosmology.berkeley.edu/inpac/CDMSCE_Jun06/ Talks/ cembranos_adddimensions.pdf.

[6] R. Kitano and Y. Nomura, "A Solution to the Supersymmetric FineTuning Problem within the MSSM," Physics Letters B, vol. 631, pp. 58-67, 2005

[7] A. Zagoździńska, K. T. Poźniak, and R. Romaniuk, "Heavy stable charged particles search by novel pattern comparator processor," Proceedings of SPIE, vol. 8454, 2012.

[8] K. T. Poźniak, "Electronics and photonics for high energy physics experiments," Proceedings of SPIE, vol. 5125, 2002.

[9] Website http://www.auger.org/.

[10] Website http://people.roma2.infn.it/ dama/web/home.html.

[11] Website http://lhc.web.cern.ch/lhc/

[12] Website http://atlas.web.cern.ch/Atlas/Collaboration/.

[13] Website http://cms.web.cern.ch/.

[14] Website http://lhcb.web.cern.ch/lhcb.

[15] The LHCb Collaboration, "The LHCb Detector at the LHC," Journal of Instrumentation, vol. 3, 2008.

[16] D. Froidevaux and P. Sphicas, "General-purpose detectors for the Large Hadron Collider," Annual Review of Nuclear and Particle Science, vol. 56, pp. 375-440, 2006.

[17] The CMS Collaboration, "The data-acquisition system of the CMS experiment at the LHC," Journal of Physics: Conference Series 331 2011, 022021.

[18] H. Spieler, "Front-End Electronics and Signal Processing," in Instrumentation in Elementary Particle Physics, AIP Conference Proceedings, 2002, vol. 674.

[19] Website http://aliceinfo.cern.ch/Public/en/Chapter2/Chap2_TOF.html.

[20] J. Christiansen, "HPTDC High Performance Time to Digital Converter," HPTDC, vol. 1.3, 2004.

[21] Website http://hepwww.rl.ac.uk/Vertex03/Talks/JornGrosseKnetter.pdf.

[22] Website http://project-slhc.web.cern.ch/project-slhc/about/.

[23] Website http://clic-study.org/.

[24] Website http://public.web.cern.ch/public/en/Research/LEPExp-en.html.

[25] Website http://www-bdnew.fnal.gov/tevatron/.

[26] The CMS Collaboration, "CMS The TriDAS ProjectTechnical Design Report, Vol.1: The Trigger Systems," CERN/LHCC 2000 - 38 CMS TDR 6.1, 2000.

[27] G. Bayatian, S. Chatrchyan, J. Krolikowski, K. Poźniak, and W. Zabołotny et al., "CMS physics technical design report, volume II: Physics performance," Journal of Physics G: Nuclear and Particle Physics, vol. 34, no. 6, 2007, index: S01.

[28] K. T. Poźniak, "FPGA-based, specialized trigger and data acquisition systems for high-energy physics experiments," Measurement Science and Technology, vol. 21, no. 6, 2010, index: 62002 .

[29] Website http://www-cdf.fnal.gov/.

[30] The CDF Collaboration, "Using Feedback to Control Deadtime in the CDF Trigger System," 2010.

[31] T. Yoshioka et al., "Upgrade of the Level-0 Trigger System for BNLE949," IEEE Transactions on Nuclear Science, vol. 51, pp. 334-339, 2004 , issue 3.

[32] I. Magrans de Abril, "The CMS Trigger Supervisor: Control and Hardware Monitoring System of the CMS Level-1 Trigger at CERN," 2008.

[33] M. Mozer, "Triggers for New Physics at the LHC," Journal of Physics. Conference Series 171 012101, 2009.

[34] R. Achenbach et al., "The ATLAS Level-1 Calorimeter Trigger," JINST, vol. 3, 2008, P03001.

[35] The ATLAS Collaboration, "Tag and Probe method for Electron ID efciencies," 2009.

[36] M. Konecki, Performance of the RPC trigger. CMS Warsaw Group, 2012.

[37] N. Neufeld, Status and Prospects of LHC Experiments Data Acquisition. CERN/PH, 2009.

[38] The CMS Collaboration, "CMS The TriDAS Project Technical Design Report, Volume 2: Data Acquisition and High-Level Trigger," CERN/LHCC 02-26 CMS TDR 6, 2002.

[39] A. Zagoździńska, R. S. Romaniuk, K. T. Pońniak, and P. Zalewski, "Tridaq systems in HEP experiments at LHC accelerator," Proceedings of SPIE, in press, 2013.

[40] R. S. Romaniuk, "Technika akceleratorowa i eksperymenty fizyk wysokich energii," Wilga 2012, Elektronika - konstrukcje, technologie zastosowania, vol. 53, no. 9, pp. 162-169, 2012
[41] R. S. Romaniuk, et al. "Optical network and FPGA/DSP based control system for free electron laser," Bulletin of the Polish Academy of Sciences, Technical Sciences, vol. 53, no. 2, pp. 123-138, 2005.

[42] R. S. Romaniuk, "Polfel - a free electron laser in Poland," Photonics Letters of Poland, vol. 1, no. 3, pp. 103-105, 2009.

[43] B. Mukherjee, et al., "Application of low-cost GaAs LEDs as kerma dosemeters and fluence monitor for high-energy neutrons," Radiation Protection Dosimetry, vol. 126, no. 1-4, pp. 256-260, 2007.

[44] R. Romaniuk, et al., "Metrological aspects of accelerator technology and high energy physics experiments," Measurement Science and Technology, vol. 18, no. 8, 2008, art.no.E01.

[45] P. Fafara, et al., "FPGA-based implementation of a cavity field controller for FLASH and X-FEL," Measurement Science and Technology, vol. 18, no. 8, pp. 2365-2371, 2008.

[46] W. Ackerman, et al., "Operation of a free-electron laser from the extreme ultraviolet to the water window," Nature Photonics, vol. 1, no. 6, pp. 336-342, 2007.

[47] T. Czarski, et al., "Superconducting cavity driving with FPGA controller," Nuclear Instruments and Methods in Physics Research A, vol. 568, no. 2, pp. 854-862, 2006.

[48] - "TESLA cavity modeling and digital implementation in FPGA technology for control system development," Nuclear Instruments and Methods in Physics Research A, vol. 556, no. 2, pp. 565-576, 2006.

[49] — , "Cavity parameters identification for TESLA control system development," Nuclear Instruments and Methods in Physics Research A, vol. 548, no. 3, pp. 283-297, 2005.

[50] R. Romaniuk, "Accelerator infrastructure in Europe EuCARD 2011," International Journal of Electronics and Telecommunications, vol. 57, no. 3, pp. 413-419, 2011.

[51] — "EuCARD 2010 accelerator technology in Europe," International Journal of Electronics and Telecommunications, vol. 56, no. 4, pp. 485488, 2010.

[52] S. Chatrchyan, et al., "The CMS experiment at the CERN LHC," Journal of Instrumentation, vol. 3, no. 8, 2008, art.no S08004.

[53] — "Commissioning of the CMS experiment and the cosmic run at four tesla," Journal of Instrumentation, vol. 5, no. 3, 2010, art.no.T03001.

[54] _ _Performance of the CMS Level-1 trigger during commissioning with cosmic ray muons and LHC beams," Journal of Instrumentation, vol. 5, no. 3, 2010, art.no.T03002.

[55] — "Performance of the CMS drift-tube chamber local trigger with cosmic rays," Journal of Instrumentation, vol. 5, no. 3, 2010 , art.no.T03003.

[56] — - "Fine synchronization of the CMS muon drift-tube local trigger using cosmic rays," Journal of Instrumentation, vol. 5, no. 3, 2010, art.no.T03004.

[57] — - "Commissioning of the CMS High-Level Trigger with cosmic muons," Journal of Instrumentation, vol. 5, no. 3, 2010, art.no.T03005.

[58] — "CMS data processing workflows during an extended cosmic ray run," Journal of Instrumentation, vol. 5, no. 3, 2010, art.no.T03006.

[59] _ - "Commissioning and performance of the CMS pixel tracker with cosmic ray muons," Journal of Instrumentation, vol. 5, no. 3, 2010, art.no.T03007.

[60] — " "Measurement of the muon stopping power in lead tungstate," Journal of Instrumentation, vol. 5, no. 3, 2010, art.no.P03007.

[61] _ _Comissioning and performance of the CMS silicon strip tracker with cosmic ray muons," Journal of Instrumentation, vol. 5, no. 3, 2010, art.no.T03008

[62] — "Alignment of the CMS silicon tracker during commissioning with cosmic rays," Journal of Instrumentation, vol. 5, no. 3, 2010 , art.no.T03009.

[63] —, "Performance and operation of the CMS electromagnetic calorimeter," Journal of Instrumentation, vol. 5, no. 3, 2010 art.no.T03010.

[64] _ - "Time reconstruction and performance of the CMS electromagnetic calorimeter," Journal of Instrumentation, vol. 5, no. 3, 2010 , art.no.T03011.

[65] — , "Performance of the CMS hadron calorimeter with cosmic ray muons and LHC beam data," Journal of Instrumentation, vol. 5, no. 3, 2010, art.no.T03012.

[66] — - "Performance of CMS hadron calorimeter timing and synchronization using test beam, cosmic ray, and LHC beam data," Journal of Instrumentation, vol. 5, no. 3, 2010, art.no.T03013.

[67] _ - "Identification and filtering of uncharacteristic noise in the CMS hadron calorimeter," Journal of Instrumentation, vol. 5, no. 3, 2010 art.no.T03014. 
[68] - "Performance of the CMS drift tube chambers with cosmic rays," Journal of Instrumentation, vol. 5, no. 3, 2010, art.no.T03015.

[69] _ "Calibration of the CMS drift tube chambers and measurement of the drift velocity with cosmic rays," Journal of Instrumentation, vol. 5, no. 3, 2010, art.no.T03016.

[70] — "Performance study of the CMS barrel resistive plate chambers with cosmic rays," Journal of Instrumentation, vol. 5, no. 3, 2010, art.no.T03017.

[71] _ - "Performance of the CMS cathode strip chambers with cosmic rays," Journal of Instrumentation, vol. 5, no. 3, 2010, art.no.T03018.

[72] - "Aligning the CMS muon chambers with the muon alignment system during an extended cosmic ray run," Journal of Instrumentation, vol. 5, no. 3, 2010, art.no.T03019.

[73] — "Alignment of the CMS muon system with cosmic-ray and beam-halo muons," Journal of Instrumentation, vol. 5, no. 3, 2010 , art.no.T03020.

[74] _ - "Precise mapping of the magnetic field in the CMS barrel yoke using cosmic rays," Journal of Instrumentation, vol. 5, no. 3, 2010 , art.no.T03021

[75] — "Performance of CMS muon reconstruction in cosmic-ray events," Journal of Instrumentation, vol. 5, no. 3, 2010, art.no.T03022.
[76] R. S. Romaniuk, "Accelerator Technology and High Energy Physics Experiments, Photonics Applications and Web Engineering, Wilga May 2012," Proceedings of SPIE, vol. 8454, 2012, art.no. 845403.

[77] _ "Physics and Plasma Research, Photonics Applications and Web Engineering, Wilga May 2012," Proceedings of SPIE, vol. 8454, 2012, art.no. 845404

[78] — , "Accelerator Science and Technology in Europe - EuCARD 2012," Proceedings of SPIE, vol. 8454, 2012, art.no. 845407.

[79] — "Proceedings of SPIE," 2011, art.no. 800802.

[80] _ - "Accelerator infrastructure in Europe: Eucard 2012," Proceedings of SPIE, vol. 8008, 2011, art.no. 800805 .

[81] _ "Free electron laser infrastructure in Europe 2012," Proceedings of SPIE, vol. 8703, 2013, art.no. 870323.

[82] _ "Accelerator science and technology in Europe - EuCARD 2012," International Journal of Electronics and Telecommunications, vol. 58, no. 4, pp. 327-334, 2012.

83] — "Space and high energy experiments - Advanced electronic systems 2012," International Journal of Electronics and Telecommunications, vol. 58, no. 4, pp. 441-462, 2012. 